\title{
ScarlETH \\ Design and Control of a Planar Running Robot
}

\section{Conference Paper}

Author(s):

Hutter, Marco (1); Remy, C.David; Hoepflinger, Mark A.; Siegwart, Roland

Publication date:

2011

Permanent link:

https://doi.org/10.3929/ethz-a-010025731

Rights / license:

In Copyright - Non-Commercial Use Permitted

Originally published in:

https://doi.org/10.1109/IROS.2011.6094504 


\title{
ScarlETH: Design and Control of a Planar Running Robot
}

\author{
Marco Hutter, C. David Remy, Mark A. Hoepflinger, and Roland Siegwart, Fellow, IEEE
}

\begin{abstract}
This paper introduces the mechanical design and the control concept of the Series Compliant Articulated Robotic Leg ScarlETH which was developed at ETH Zurich for fast, efficient, and versatile locomotion. Inspired by biological systems, we seek to achieve this through large compliances in the joints which enable natural dynamics, allow temporary energy storage, and improve the passive adaptability. A sophisticated chain and cable pulley design minimizes the segment masses, places the overall CoG close to the hip joint, and maximizes the range of motion. Nonlinearities in the damping and an appropriate low-level controller allow for precise torque control during stance and for fast task space position control during swing. This paved the road for the combined application of a virtual model controller for ground contact and a modified Raibert style controller for flight phase which was successfully tested in planar running.
\end{abstract}

\section{INTRODUCTION}

C YURRENT legged systems can be roughly separated into two distinct classes [1]: Simplistic devices, often with many degrees of under-actuation, use inertia driven movements and springs to propel themselves forward. They create remarkable abilities with respect to minimal control effort [2, 3], high locomotion speed [4, 5], and robustness [6]. These systems are often inspired by biology and, in return, can be used to study and understand human or animal locomotion principles [7-9].

In contrast, more standard robotic systems which are built on the long-time experience of kinematically controlled manipulators, have presented great robustness and versatility. For example, in the field of humanoid robots, systems can carefully select their footholds [10] or handle objects [11]. For quadrupedal robots, the DARPA little-dog challenge [12] demonstrated the state of the art considering footstep planning in highly unstructured terrain, and Boston Dynamic's BigDog [13] sets the standard for fast and robust (yet highly inefficient) locomotion. Towards the same direction, our research group developed a small quadruped [14] that, to the best of our knowledge, exhibits currently the highest mobility in terms of kinematic range of motion.

The missing link to combine these two approaches into one unified platform is an appropriate actuator that allows precise torque and position control for versatile motions, and enables passive dynamic motions for fast and dynamic locomotion such as running or hopping. One particularly

Manuscript received March 14, 2011. This work was supported in part by the Swiss National Science Foundation (SNF) (project 200021_119965/1), the Swiss National Centre of Competence in Research (NCCR), and the Hans-Eggenberger-Foundation Marco Hutter, C. David Remy, Mark Hoepflinger, and Roland Siegwart are with the Autonomous Systems Lab, Institute of Robotics and Intelligent Systems, Swiss Federal Institute of Technology (ETHZ), Zurich, Switzerland (+41 4463274 17; mahutter@ethz.ch, cremy@ethz.ch, markho@ethz.ch, rsiegwart@ethz.ch)

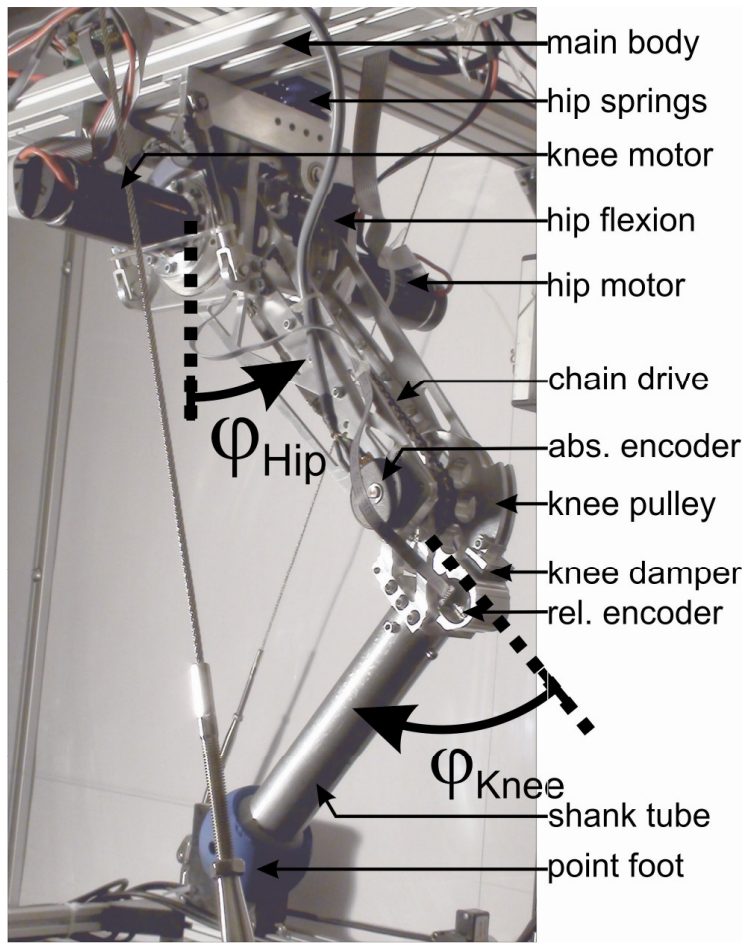

Fig. 1. ScarlETH, the Series Compliant Articulated Robotic Leg combines versatility, speed, and efficiency.

promising approach to close this gap is Series Elastic Actuation (SEA), especially with respect to efficiency, size, and indoor applicability. While such actuators were originally designated solely as torque-controllable elements that also protect the gearbox from impact collisions $[15,16]$, their use can be extended to additionally serve as structural dynamic elements: Just as in nature, where elastic energy is stored in muscles and tendons $[9,17]$ (this can be around $50 \%$ of the whole mechanical energy in human running), compliant elements can be used in robots to temporarily store energy and shape the natural dynamics of the system. This substantially increases the overall performance with regard to efficiency and speed. Examples that implement this concept include Marc Raibert's early robots [18, 19], which used air as compliant element, and more recent quadrupedal [20] or single legged [21-25] platforms.

In our research we extended the principle of high compliance series elastic actuation to include nonlinear spring and damping characteristics as well as sophisticated lowlevel control to enable precise position control, high fidelity torque control, and natural dynamic motions with one single piece of hardware. In particular, this paper builds up to successful 2D running experiments with the articulated robotic leg ScarlETH: It introduces the mechanical design and highlights features that allow combining natural dynamics 
with high versatility, it explains the low-level control setup for torque and position control, and it shows how virtual model control (during ground contact) and a modified Raibert style controller (during flight) can be combined to create an efficient running motion. The paper is accompanied by two short videos that illustrate the mechanical setup and the presented methods and results.

\section{MeChatronic SYSTEM}

ScarlETH (Fig. 1, Fig. 2) consists of three segments: A main body, a thigh, and a shank. They are connected at the hip and the knee with joints driven by high compliant series elastic actuators. A miniature chain and cable pulley system (Fig. 2) allows placing both actuators (for hip and knee flexion/extension) directly at the hip joint. The overall center of gravity (CoG) is thus positioned close to the hip axis and the inertia of the leg segments was kept minimal. This is beneficial for two reasons: It facilitates fast leg motion with high accelerations and reduces the energy losses in the impact collisions at touch down [26]. This configuration achieves a very large range of motion: the leg can be fully retracted in the knee $\left(\varphi_{\text {Knee }} \in\left\{-90^{\circ}, 170^{\circ}\right\}\right)$, and can swing $\left(\varphi_{\text {Hip }} \in\left\{-85^{\circ},+85^{\circ}\right\}\right)$ around the hip axis. Strong DCmotors in combination with 1:80 harmonic drive gears provide up to $30 \mathrm{Nm}$ torque per joint. All motors and gears are tightly packed into one single mechanical component that simultaneously serves as housing for the gear-box, holds the bearings of the chain-drives, and functions as hip axis. Due to this high integration, we were able reduce the weight of the overall flexion extension drive by a factor of two in comparison to a solution [27] using off-the-shelf gearboxes with housing. The total weight of the leg is about $3.0 \mathrm{~kg}$ (Fig. 2), and it currently carries $3.3 \mathrm{~kg}$ of payload (a guiding unit that restricts the motion of the leg to $2 \mathrm{D}$ constitutes most of this weight). With segment lengths of $0.2 \mathrm{~m}$ the final prototype is compact, lightweight and can thus be handled safely by a single person, yet is strong enough to carry various sensor units. To protect the system from crashes, the miniature chain drive is designed to be the weakest part for the knee and the hip actuation with a breaking torque that is about $20 \%$ below the peak torque the gearboxes can sustain. In case of failure, the chain can be replaced within $5 \mathrm{mi}-$ nutes.

One of the requirements for high efficiency is that the actuator should only provide positive power and should never remove energy from the system. For running (which is similar to a springy bouncing motion [17, 28, 29]), the necessary fluctuations in the systems energy content can be achieved by storing energy in elastic elements during the deceleration phase after touch-down and releasing it in the subsequent acceleration phase before lift-off. The more compliant the elastic elements are, the longer this conversion of energy takes, and the longer the stance duration of the system is. This allows for longer actuator action, enables us to create the same amount of energy with less powerful actuators, and hence enables the usage of smaller and more lightweight actuators [29, 30].

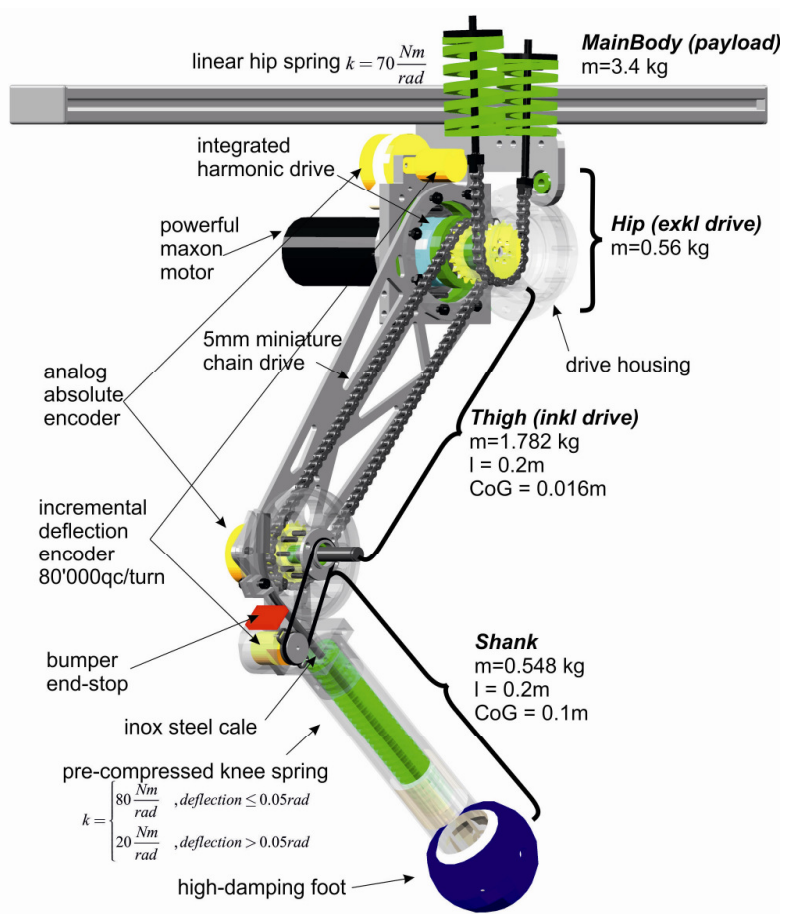

Fig. 2. System description including a visualization of the series elastic actuation principle for hip and knee flexion/extension.

These ideas are realized in hardware with a highly compliant spring in the knee joint. It has a stiffness of $20 \mathrm{Nm} / \mathrm{rad}$ and can undergo deflections of up to $90^{\circ}$ during stance. Approximately $30 \mathrm{~J}$ of energy can be stored in this spring, which corresponds to a vertical excursion of the total systems mass by $0.5 \mathrm{~m}$ and an equivalent hopping height. Precompression of all springs in combination with the harmonic drives makes the whole system backlash free.

The fully actuated system is operable in torque or position/velocity mode. Incremental sensors in all motors and joints measure the spring deflections with an accuracy of 80 '000 counts per revolution and the motor position with 160 '000 counts. Additional absolute sensors monitor the joint angles and are used for safety and initialization purposes. Each motor (Maxon RE35, 90W) is controlled by an individual servo controller that is responsible for current $(10 \mathrm{kHz})$ as well as motor velocity control $(1 \mathrm{kHz})$. These controllers, as well as the incremental and analog sensor data acquisition boards are connected with a CAN-bus interface to a National Instruments compactRIO (reconfigurable I/O) that combines an FPGA-controller and a real time processor with the different layers of data acquisition, torque control, and high level control. The position and torque control loops are implemented on the FPGA $(500 \mathrm{~Hz})$ while the high level controllers (e.g. Raibert flight controller, virtual model stance controller) are running on the real time processor $(250 \mathrm{~Hz})$. In the current single leg design, energy supply is provided off-board.

\section{CONTROL StRATEGIES}

The goals of versatility, speed, and efficiency seem to be contradictory when it comes to control [1]. As stated above, efficiency and speed can only be achieved with high com- 


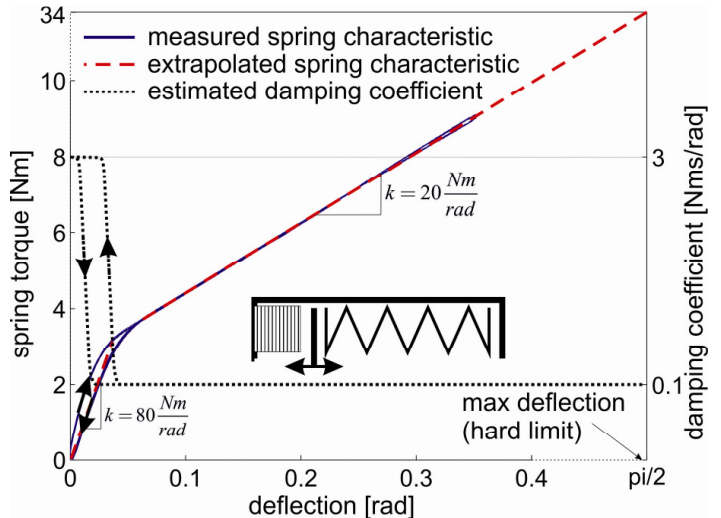

Fig. 3. The pre-compressed spring characteristic in the knee joint allows for efficient energy storage and release during stance $\Delta \varphi>0.05$, but enforces zero deflection during non-contact phase.

pliances as they are needed for temporary energy storage and to generate the desired natural dynamics in the mechanical system. However, strong elasticities also create undesired oscillations which make quick and precise foot-placement difficult. This issue is described in detail in [27], were we refer to it as the damping dilemma. This is why today's most versatile systems rely on high precision kinematic control and are implemented on mechanically rigid systems. In the following we show how it can be alleviated through appropriate design of the mechanical system and a suitable controller, and how both, precise position and precise torque control, can be achieved.

\section{A. Position Control}

Due to the low inertia of the shank segment, the eigenfrequency of the knee dynamics is high and active damping would require a closed-loop control system in a bandwidth range that is not applicable on our hardware. In previous work, we tackled this problem by using a nonlinear precompressed knee-spring that exploited internal collisions to significantly increase the damping while the leg was not on the ground [27]. This concept was developed further and simplified in the current design: A pre-loaded compression spring in series with a unilateral damper shows the springdamping characteristic of Fig. 3. During the stance phase, when large knee torques deflect the spring by more than 0.05 rad, torque control can be performed in the linear, lowdamping region ( $c_{\text {spring }}, d_{\text {spring }}$ ), while the unloaded leg (deflection $<0.05 \mathrm{rad}$ ) has high stiffness as well as high damping properties $\left(c_{\text {damper }} d_{\text {damper }}\right)$ that passively attenuate undesired deflections.

This means that in order to generate a desired knee joint angle while the leg is in the air, position control of the kneemotor is sufficient. The joint will follow closely. During flight, torques in the joint only occur due to acceleration of the small inertia of the shank and are too small to create noticeable deflections in the high-stiffness/high-damping range of the knee spring. Fig. 4a shows that for step inputs in both directions minimal deflections in the region of maximal positive velocity occurs (A, B). Initial joint deflections (Fig. 4b) are damped out with no overshoot (C). The mechanical damping quickly attenuates any undesired deflec-
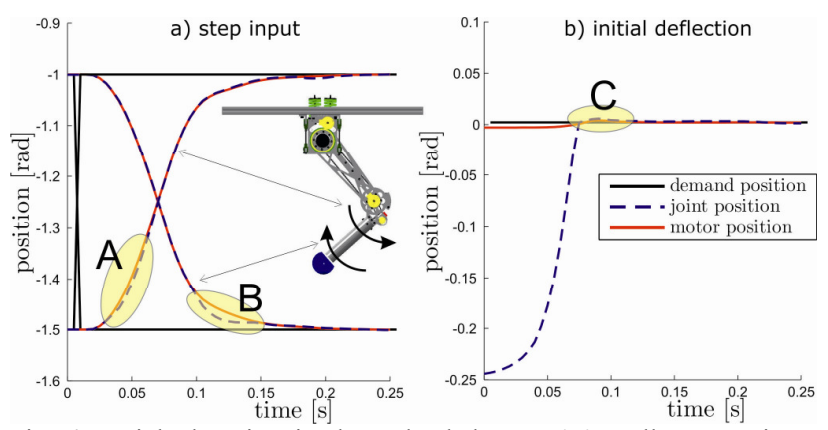

Fig. 4. High damping in the unloaded knee joint allows precise position control with minimal deflection for demand step inputs (a) and nearly no overshoot for initial joint deflections (b).

tion and thus allows for precise position control during flight.

Since at the hip joint elastic deflections in both directions are required, the mechanical concept of the knee joint is not applicable. The low natural damping of the steel springs is insufficient for fast and accurate position control and the system must be damped actively. To this end, we implemented an LQG controller based on the position and speed measurements of motor and joint (Fig. 5). Analyzing the identified spring/damper and leg dynamics shows the expected $2^{\text {nd }}$ order mass-spring oscillator behavior with a large peak at the eigenfrequency $(7.5 \mathrm{~Hz}, 18 \mathrm{~dB})$ and a cut-off frequency of about $10 \mathrm{~Hz}$. The controlled motor/gearbox dynamics $\frac{\dot{\varphi}_{\text {mot }}}{\dot{\varphi}_{\text {des }}}$ shows a low-pass characteristic with a bandwidth of about $100 \mathrm{~Hz}$ [31] such that the mass-spring oscillator is the clearly dominating dynamics. For the position controller design, the plant (velocity controlled motor $\frac{\varphi_{g b}}{\dot{\varphi}_{\text {des }}}$, spring-mass oscillator $\frac{\varphi_{\text {joint }}}{\varphi_{g b}}$ ) can be largely simplified to an integrator in series with a $2^{\text {nd }}$ order system. Having accurate measurements of the motor and joint states at hand, a direct LQG implementation without observer can be designed and implemented. The responses to step inputs and initial spring deflections show a remarkable improvement in comparison to the standard motor position control (Fig. 6a). Simulation and experiment for both tests are congruent, including saturation limitations. The position bandwidth (Fig. 6b) for small amplitudes is about $9 \mathrm{~Hz}$ (limited by the mechanical lowpass filter) and reduced for higher amplitudes due to actuator saturation effects. With a maximal complementary sensitivity of $T_{\max }<1 d B$, the spring dynamics are well damped.

\section{B. Torque control}

Sophisticated control algorithms (such as [32-34]) that can cope with robot ground interaction require the possibility of precise joint torque control. With this, it is also possible to lower the gains on the position feedback loop and rely mostly on (model based) feed-forward joint torques. This can greatly improve the overall robustness in unknown terrain [12]. While most standard applications are based either on inaccurate current measurements in combination with some 


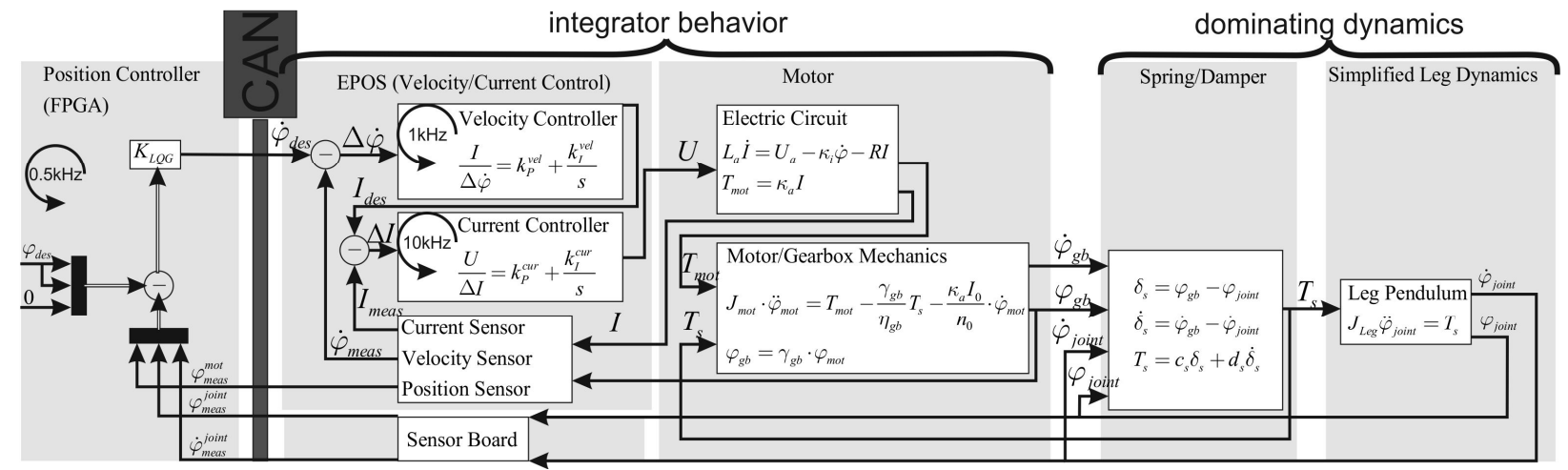

Fig. 5. LQG position control scheme for the hip joint. Since the natural damping is too low to control only the actuator side, a full state feedback LQG controller is implemented based on a simplified controlled motor dynamics model and linearized leg dynamics.

kind of gearbox model or expensive (and impact sensitive) torque/force sensors, the series elasticity allows very precise torque regulation through control of the spring deflection. Identification of the hip spring $[-20 \mathrm{Nm},+20 \mathrm{Nm}]$ and the (loaded) knee spring $\left[T_{\text {Pre-Compression }},+30 \mathrm{Nm}\right]$ has shown perfect linearity and minimal damping. Thus regulating the deflection is appropriate for precise torque control. With a simple PI controller on joint deflection and a joint disturbance compensator in a cascaded velocity based control structure (Fig. 5) we were able to fulfill good tracking performance (tested in step inputs) and joint disturbance rejection (zero torque test while joint is disturbed) [31]. The closed loop torque control bandwidth is about $28 \mathrm{~Hz}$ for low and decreasing to $11 \mathrm{~Hz}$ for higher amplitudes. The disturbance compensator allows for a very low integrator gain in the feedback loop which prevents overshoot and increases robustness (phase margin $>85^{\circ}$ ).

\section{2D RUNNING}

For many animals (including bipeds, quadrupeds, and insects), the biomechanics of running strongly resembles the dynamics of the so called Spring Loaded Inverted Pendulum (SLIP) template $[35,36]$. While moving forward, their center of gravity is moving as if it was oscillating on a single spring that acts during ground contact between the $\mathrm{CoG}$ and the center of pressure in the feet. The CoG pivots around the $\mathrm{CoP}$, such that the horizontal action of this virtual spring is symmetric and does not impact the forward velocity. The dynamics are characterized by a periodic exchange of potential, kinetic, and elastic energy. The template has the valuable property that the vertical bouncing motion (characterized by a spring stiffness and hopping height) can be decoupled entirely from the forward motion (which is characterized by the angle of attack). It is straight forward to use this template as a base for control algorithms of prismatic hoppers $[18,24]$.

\section{1) Virtual Model Control}

The mechanical design of ScarlETH closely matches the properties of the SLIP template: the robot is designed with lightweight low inertia segments, has a point foot, and the global center of mass is located near the hip joint. However, due to the articulated design, the elastic rebound and the angle of attack are coupled in hip and knee and must be decoupled in the controller. To this end, we introduce a virtual force element (with force $\mathbf{F}_{\text {virtual }}$ ) that acts between the foot of the robot and the hip; that is, approximately along the line CoP-CoG, as it is required for the SLIP model. Using the kinematics of the leg, the joint torques $\mathbf{T}$ can be computed such that they create the same effect as the virtual force element [32]:

$$
\begin{gathered}
\mathbf{T}=\mathbf{J}^{T} \mathbf{F}_{\text {virtual }} . \\
\mathbf{J}=\frac{\partial \mathbf{r}_{H i p}-\partial \mathbf{r}_{F o o t}}{\partial \mathbf{q}_{r}}
\end{gathered}
$$

While both, the base $\mathbf{r}_{\text {Hip }}(\mathbf{q})$ and foot $\mathbf{r}_{\text {Foot }}(\mathbf{q})$ positions are functions of $\mathbf{q}=\left[\mathbf{q}_{h}, \mathbf{q}_{r}\right]$ with unactuated hip coordinates $\mathbf{q}_{h}$ and joint coordinates $\mathbf{q}_{r}$, their difference and hence the relative Jacobian $\mathbf{J}\left(\mathbf{q}_{r}\right)$ is solely a function of the joint configuration. In the most basic implementation, the virtual force $\mathbf{F}_{\text {virtual }}$ is simply calculated as a linear spring (-damper) element between foot contact point and hip joint or CoG. In this paper, a modified implementation is presented that demonstrated an excellent experimental behavior. For this, the virtual force was decomposed into a vertical and a horizontal component: The virtual force in $y$-direction provides the springy behavior:

$$
\mathbf{F}_{\text {virtual }}^{y}=c_{y}\left(y_{0}-\left(y_{\text {Hip }}-y_{\text {Foot }}\right)\right)+d_{y}\left(-\dot{y}_{\text {Hip }}\right)+g_{y}
$$

with the spring stiffness $c_{y}$, the damping coefficient $d_{y}$ and a gravity compensation term $g_{y}$. The computation requires only relative position/velocity information of the hip against the contact point and can hence be performed using only internal joint sensors. In contrast to the SLIP template, which assumes a conservative system, in the actual robot energy is lost in the collision at every instant of touch-down. This loss is invisible for the controller and the closed loop system would not be energetically conservative. To compensate the loss and add energy to the system, the zero spring length $y_{0}$ is increased from the value measured at the instance of touchdown [37]. This is equivalent to virtually pre-compressing the spring and releasing the stored energy during stance:

$$
y_{0}=y_{\text {landing }}+\Delta y
$$




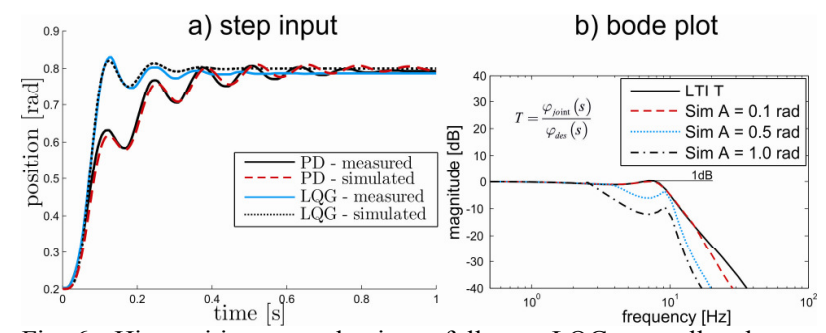

Fig. 6. Hip position control using a full state LQG controller shows remarkable better performance than standard actuator control (a) with a bandwidth of about $9 \mathrm{~Hz}$ for small amplitudes.

Depending on this virtual lengthening $\Delta y$, the apex height that is reached during hopping can be changed. By increasing $\Delta y(4)$ or increasing $d_{y}$ (3), energy can be introduced respectively removed from the system allowing us to start and stop running within a single step.

The forward motion is controlled during stance by modulation of the virtual force in $\mathrm{x}$-direction. This allows fast acceleration and deceleration in horizontal direction, independent of the leg position. A simple proportional position controller is used to this end:

$$
F_{\text {virtual }}^{x}=\operatorname{sat}\left(k_{x}\left(x_{\text {des }}-x_{\text {Base }}\right)\right) \in\left[-F_{\text {neg }}^{x}, F_{p o s}^{x}\right]
$$

The force limitations described by the saturation function sat ( ) are chosen such that excessive action (which could result in tipping over) is avoided. Controlling the absolute position in $\mathrm{x}$-direction requires measurement of the actual horizontal position. In the presented prototype, this is done by an external sensor mounted at the guiding unit. The current test setup with a blocked pitch angle, requires no stabilization of the main body orientation. For future work, this could be included in the same framework by adding a virtual force on the main body orientation $F_{\text {virtual }}^{\theta}=f\left(\theta, \theta_{\text {desired }}\right)$. This would additionally require the possibility for absolute pitch angle measurement.

\section{2) Modified Raibert flight control}

A modified Raibert style [18] controller is applied during flight phase. Due to the articulated design, the standard controller [18] that was developed for prismatic legs has to be augmented by an offset angle $\alpha_{0}$ to compensate for the coupling effects of thigh and shank as well as to compensate for the horizontal impact happening at landing:

$$
\alpha\left(\dot{x}_{\text {Base }}\right)=k_{F F} \cdot \dot{x}+k_{F B} \cdot\left(k_{\text {Pos }}\left(x_{\text {des }}-x\right)-\dot{x}\right)+\alpha_{0}
$$

whereby $k_{F F}>0$ corresponds to the Raibert feedforward, $k_{F B}<0$ the feedback, $k_{P o s}$ to the position gain. Since we are already applying a horizontal force during stance phase to control the forward velocity, horizontal position control works also without the speed term $\left(k_{F B}=0\right)$.

\section{3) Results}

The presented 2D hopping control structure showed great stability in simulation and experiment. The desired position in x-direction was achieved within a few steps (Fig. 7), showing nearly no overshoot at the target position. The

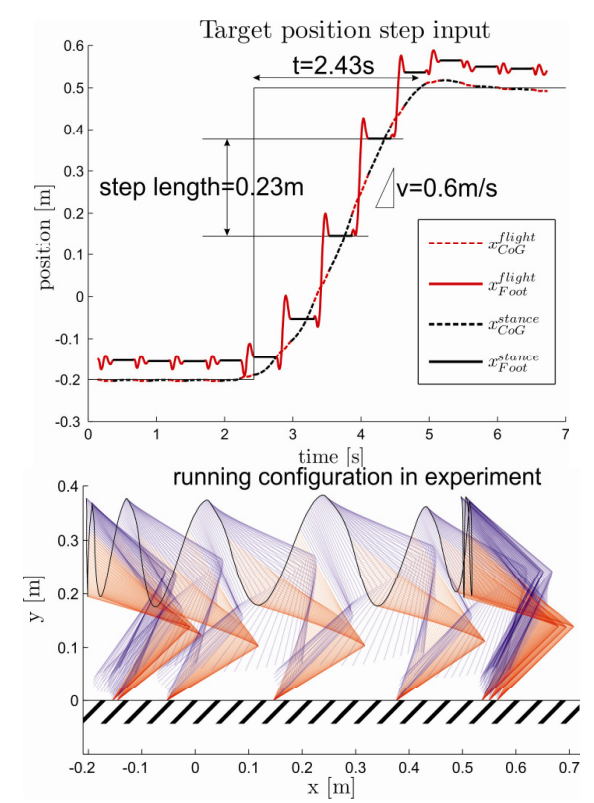

Fig. 7. A horizontal demand position is reached within only a few steps. The step length in the top speed region is about $0.23 \mathrm{~m}$, the maximal speed at this position is about $0.6 \mathrm{~m} / \mathrm{s}$.

maximal step length was $0.23 \mathrm{~m}$ at a speed of $0.6 \mathrm{~m} / \mathrm{s}$. Reaching even higher velocities was limited by the length of the test bench. Speed and step length could be adjusted by modifying the maximal horizontal forces $F_{n e g}^{x}, F_{p o s}^{x}$. The hopping height in the example was about $0.08 \mathrm{~m}$ at apex transit. The presented joint controller was fast enough to achieve the desired impact configuration shortly after apex transit. Further on, the virtual model stance control with the bouncing behavior in y-direction resulted in a $\mathrm{CoG}$ trajectory that closely matched its equivalent SLIP template.

\section{CONCLUSION AND OUTLOOK}

This paper introduced ScarlETH, a series compliant articulated robotic leg for fast, efficient, and versatile applications. It is lightweight, has low segment inertia, powerful actuation, and an easy-to-handle size. The system is tightly integrated, exhibits an extensive range of motion, and is extremely robust. What sets it apart from comparable devices is that the leg can be operated in full position or full torque controlled mode. The sophisticated mechanical and controller design allows using high compliance in the knee to increase efficiency and speed but, at the same time, to do precise and fast position control as it is needed for robust locomotion in rough terrain.

Position and torque control showed more than satisfactory performance in joint and task space control tests. A virtual model controller for the stance phase in combination with a modified Raibert style flight controller allowed controlled forward and backward hopping. Within a few steps and with a speed of about $0.6 \mathrm{~m} / \mathrm{s}$ a desired horizontal position was reached. The jumping height was adjusted by changing the damping and/or the neutral virtual spring position. The virtual ground stiffness was tuned in experiments such that 
the actual torque in the knee could be provided to a large degree by the passive dynamics of the knee spring [31]. In the current test-setup, the leg is limited to a hopping range of $\pm 0.5 \mathrm{~m}$.

The leg design is currently extended with an additional degree of freedom for hip abduction/adduction. This joint will feature the same SEA design and controller architecture. The complete leg is used in a middle-sized (linear dimensions around $0.70 \mathrm{~m}$ ) quadruped robot that unites the three goals of versatility, efficiency, and speed into one platform. The final prototype should be able to walk in unstructured terrain using full torque control, do precise foot positioning using high gain task space position control, and exploit elastic energy storage for high dynamic maneuvers.

\section{REFERENCES}

[1] A. D. Kuo, "Choosing Your Steps Carefully: Trade-offs between economy and versatility in dynamic walking bipedal robots," IEEE Robotics \& Automation Magazine, vol. 14, pp. 18-29, June 2007.

[2] F. Iida, G. Gomez, and R. Pfeifer, "Exploiting body dynamics for controlling a running quadruped robot," in ICRA, Barcelona, Spain, 2005, pp. 229-35.

[3] S. Rutishauser, A. Sprowitz, L. Righetti, and A. J. Ijspeert, "Passive compliant quadruped robot using Central Pattern Generators for locomotion control," in Biomedical Robotics and Biomechatronics, 2008. BioRob 2008. 2nd IEEE RAS \& EMBS International Conference on, 2008, pp. 710-715.

[4] D. Papadopoulos, "STABLE RUNNING FOR A QUADRUPED ROBOT WITH COMPLIANT LEGS," in Department of Mechanical Engineering. vol. PhD Montreal, Canada: McGill University, 2000.

[5] J. A. Smith and I. Poulakakis, "Rotary gallop in the untethered quadrupedal robot scout II," in Intelligent Robots and Systems, 2004. (IROS 2004). Proceedings. 2004 IEEE/RSJ International Conference on, 2004, pp. 2556-2561 vol.3.

[6] R. Altendorfer, U. Saranli, H. Komsuoglu, D. Koditschek, H. B. Brown, M. Buehler, N. Moore, D. McMordie, and R. Full, "Evidence for spring loaded inverted pendulum running in a hexapod robot," Experimental Robotics Vii, vol. 271, pp. 291-302, 2001.

[7] R. M. Alexander, Principles of Animal Locomotion. Princeton, NJ: Princeton University Press, 2003.

[8] R. M. Alexander, "Simple models of human locomotion." vol. 1: Taylor \& Francis, 1997, pp. $129-135$.

[9] M. H. Dickinson, C. T. Farley, R. J. Full, M. A. R. Koehl, R. Kram, and S. Lehman, "How Animals Move: An Integrative View," Science, vol. 288, pp. 100-106, April 72000.

[10]Y. Sakagami, R. Watanabe, C. Aoyama, S. Matsunaga, N. Higaki, and K. Fujimura, "The intelligent ASIMO: system overview and integration," in International Conference on Intelligent Robots and System (IROS), Lausanne, Switzerland, 2002, pp. 2478-2483.

[11]C. Ott, O. Eiberger, W. Friedl, B. Bauml, U. Hillenbrand, C. Borst, A. Albu-Schaffer, B. Brunner, H. Hirschmuller, S. Kielhofer, R. Konietschke, M. Suppa, T. Wimbock, F. Zacharias, and G. Hirzinger, "A Humanoid Two-Arm System for Dexterous Manipulation," in Humanoid Robots, 2006 6th IEEE-RAS International Conference on, 2006, pp. 276-283.

[12]M. Kalakrishnan, J. Buchli, P. Pastor, M. Mistry, and S. Schaal, "Fast, Robust Quadruped Locomotion over Challenging Terrain," in ICRA 2010, Anchorage, AK, 2010, p. in Press.

[13]M. Buehler, R. Playter, and M. H. Raibert, "Robots Step Outside," in International Symposion of Adaptive Motion of Animals and Machines Illmenau, Germany, 2005.

[14]C. D. Remy, O. Baur, M. Latta, A. Lauber, M. Hutter, M. H. Hoepflinger, C. Pradalier, and R. Siegwart, "Walking and Crawling with ALoF - A Robot for Autonomous Locomotion On Four Legs," in International Conference on Climbing and Walking Robots CLAWAR, Nagoya, Japan, 2010.

[15]G. Pratt and M. Williamson, "Series elastic actuators," in IROS, Pittsburg, PA, 1995, pp. 399-406.
[16]D. W. Robinson, J. E. Pratt, D. J. Paluska, and G. A. Pratt, "Series elastic actuator development for a biomimetic walking robot," in International Conference on Advanced Intelligent Mechatronics (AIM), Atlanta, USA, 1999, pp. 561-568.

[17] G. A. Cavagna, N. C. Heglund, and C. R. Taylor, "Mechanical work in terrestrial locomotion: two basic mechanisms for minimizing energy expenditure," AJP Regul Integr Comp Physiol, vol. 233, pp. 243-261, November 11977.

[18]M. H. Raibert, Legged robots that balance. Cambridge, Mass.: MIT Press, 1986.

[19]M. H. Raibert, "Trotting, Pacing and Bounding by a Quadruped Robot," Journal of Biomechanics, vol. 23, pp. 79-98, 1990.

[20] J. G. Nichol, S. P. N. Singh, K. J. Waldron, L. R. Palmer, III, and D. E. Orin, "System Design of a Quadrupedal Galloping Machine." vol. 23, 2004, pp. 1013-1027.

[21]J. W. Hurst, "The Role and Implementation of Compliance in Legged Locomotion," in Robotics Institute. vol. PhD Pittsburgh, PA: Carnegie Mellon University, 2008, p. 110.

[22] S. Curran and D. E. Orin, "Evolution of a jump in an articulated leg with series-elastic actuation," in IEEE International Conference on Robotics and Automation (ICRA) Pasadena, CA, USA, 2008, pp. 252258.

[23] S. H. Hyon and T. Mita, "Development of a biologically inspired hopping robot-"Kenken"," in Robotics and Automation, 2002. Proceedings. ICRA 'O2. IEEE International Conference on, 2002, pp. 3984-3991 vol.4.

[24]P. Gregorio, M. Ahmadi, and M. Buehler, "Design, control, and energetics of an electrically actuated legged robot," IEEE Transactions on Systems Man and Cybernetics. Part B: Cybernetics, vol. 27, pp. 626-634, Aug. 1997.

[25]H. De Man, D. Lefeber, and J. Vermeulen, "Design and Control of a Robot with One Articulated Leg for Locomotion on Irregular Terrain," in CSIM courses and lectures. vol. 405, A. Morecki, G. Bianchi, and M. Wojtyra, Eds.: SpringerWienNewYork, 1998, pp. 417-424.

[26]J. P. Schmiedeler and K. J. Waldron, "Impact analysis as a design tool for the legs of mobile robots," Advances in Robot Kinematics, pp. 129$136,442,2000$.

[27]M. Hutter, C. D. Remy, and R. Siegwart, "Design of an Articulated Robotic Leg with Nonlinear Series Elastic Actuation," in International Conference on Climbing and Walking Robots CLAWAR, Istanbul, Turkey, 2009.

[28] G. A. Cavagna, F. P. Saibene, and R. Margaria, "Mechanical work in running," J Appl Physiol, vol. 19, pp. 249-256, March 1, 19641964.

[29]C. T. Farley, J. Glasheen, and T. A. Mcmahon, "Running Springs Speed and Animal Size," Journal of Experimental Biology, vol. 185, pp. 71-86, Dec 1993.

[30]A. Seyfarth, H. Geyer, M. Gunther, and R. Blickhan, "A movement criterion for running," Journal of Biomechanics, vol. 35, pp. 649-655, May 2002.

[31]M. Hutter, C. D. Remy, M. H. Hoepflinger, and R. Siegwart, "High Compliant Series Elastic Actuation for ScarlETH," in International Conference on Climbing and Walking Robots CLAWAR 2011 Paris, Fr, 2011.

[32]J. Pratt, C. M. Chew, A. Torres, P. Dilworth, and G. Pratt, "Virtual model control: An intuitive approach for bipedal locomotion," International Journal of Robotics Research, vol. 20, pp. 129-143, Feb 2001.

[33]L. Sentis, "Compliant Control of Whole-Body Multi-Contact Behaviors in Humanoid Robots," in Motion Planning for Humanoid Robots: Springer Global Editorial, 2009.

[34]M. Mistry, J. Buchli, and S. Schaal, "Inverse Dynamics Control of Floating Base Systems Using Orthogonal Decomposition," 2010 Ieee International Conference on Robotics and Automation (Icra), pp. 3406$3412,5626,2010$.

[35]R. M. Alexander, "3 Uses for Springs in Legged Locomotion," International Journal of Robotics Research, vol. 9, pp. 53-61, April 1990.

[36]R. Blickhan, "The spring-mass model for running and hopping," Journal of Biomechanics, vol. 22, pp. 1217-1227, 1989.

[37]M. Hutter, C. D. Remy, M. H. Hoepflinger, and R. Siegwart, "SLIP Running with an Articulated Robotic Leg," in International Conference on Intelligent Robots and Systems, Taipei, Taiwan, 2010. 